\title{
Study on Ship Motions and Capsizing in Following Seas (Final Report)
}

\author{
by Masami Hamamoto*, Member, Yoonsoo Kim**, Member \\ Katsuya Uwatoko**
}

\begin{abstract}
Summary
Critical situations leading up to capsize are pointed out for a ship in severe following and quartering seas by using the aid of numerical simulation program. The effect of several parameters on the critical situation is discussed to investigate the possible causes of capsizing. In addition, Froude-Krylov force acting on a ship with large amplitude displacement are evaluated in the practical formulas to make simulation program simple.
\end{abstract}

\section{Introduction}

When a ship is running in severe following and quartering seas, it is well known that the ship may come across a dangerous situation leading up to capsize and the situation may be affected by a wide variety of causes like pure loss of stability, parametric resonance and broaching-to as Paulling 13) pointed out. At present it will be difficult to obtain a complete solution of them because there are some problems to be solved for extreme motion both ship and waves. However, approximate approaches have been tried from the following point of views.

In following sea condition the encounter frequency of ship to waves is in a low frequency range and then the hydrostatic and hydrodynamic forces acting on her are slowly changed with respect to the relative position of ship to waves, thus the hydrostatic forces including Froude-Krylov forces are considered to play a significant role for the ship motion of large amplitude.

Such a property gives some informations to an analytical study on the ship motion and capsizing in severe following seas as reported in the previous papers.

In this final paper the authers would like to summarize the important points of this study and to add something new. First one is to obtain practical formulas for the evaluation of Froude-Krylov forces and moments acting on the instantaneous immersed surface of the ship varied with respect to the relative position of ship to waves. Second one is to find out a critical situation leading up to capsize of the ship in severe

* Department of Naval Architecture and Ocean Engineering, Osaka University

** Graduate School, Department of Naval Architecture and Ocean Engineering, Osaka University following and quartering seas by using the aid of a mathematical model which can carry out time domain numerical simulations.

\section{Practical formula of Froude-Krylov forces and restoring force}

In general, Froude-Krylov forces acting on the immersed surface of a ship are computed under the assumption where the motions both ship and waves are linearized and the practical formulas are obtained from the so-called strip method. But, in case of extreme motions both ship and waves, it is necessary for the evaluation of Froude-Krylov forces to take into account a nonlinear effect of the ship hull shape as the immersed surface of a ship varies largely with respect to the relative position of ship to waves. For this requirement, an analytical approach is tried here to obtain the practical formulas.

When a ship is displaced in the earth axes $O-\xi, \eta, \zeta$ as shown in Fig. 1, as well known, the components $X_{(F, K)}, Y_{(F, K)}, Z_{(F, K)}, K_{(F, K)}, M_{(F, K)}$ and $N_{(F . K)}$ of FroudeKrylov forces and moments in the body axes, $G-x, y, z$, are

Froude-Krylov forces

$$
\begin{aligned}
X_{(F, K)} & =-\iiint_{V} \frac{\partial p}{\partial x} d V \\
Y_{(F, K)} & =-\iiint_{V} \frac{\partial p}{\partial y} d V \\
Z_{(F . K)} & =-\iiint_{V} \frac{\partial p}{\partial z} d V
\end{aligned}
$$

Froude-Krylov moments

$$
\begin{aligned}
& K_{(F . K)}=-\iiint_{V}\left[\left(\frac{\partial p}{\partial z}\right) y-\left(\frac{\partial p}{\partial y}\right) z\right] d V \\
& M_{(F . K)}=-\iiint_{V}\left[\left(\frac{\partial p}{\partial x}\right) z-\left(\frac{\partial p}{\partial z}\right) x\right] d V \\
& N_{(F . K)}=-\iiint_{V}\left[\left(\frac{\partial p}{\partial y}\right) x-\left(\frac{\partial p}{\partial x}\right) y\right] d V
\end{aligned}
$$



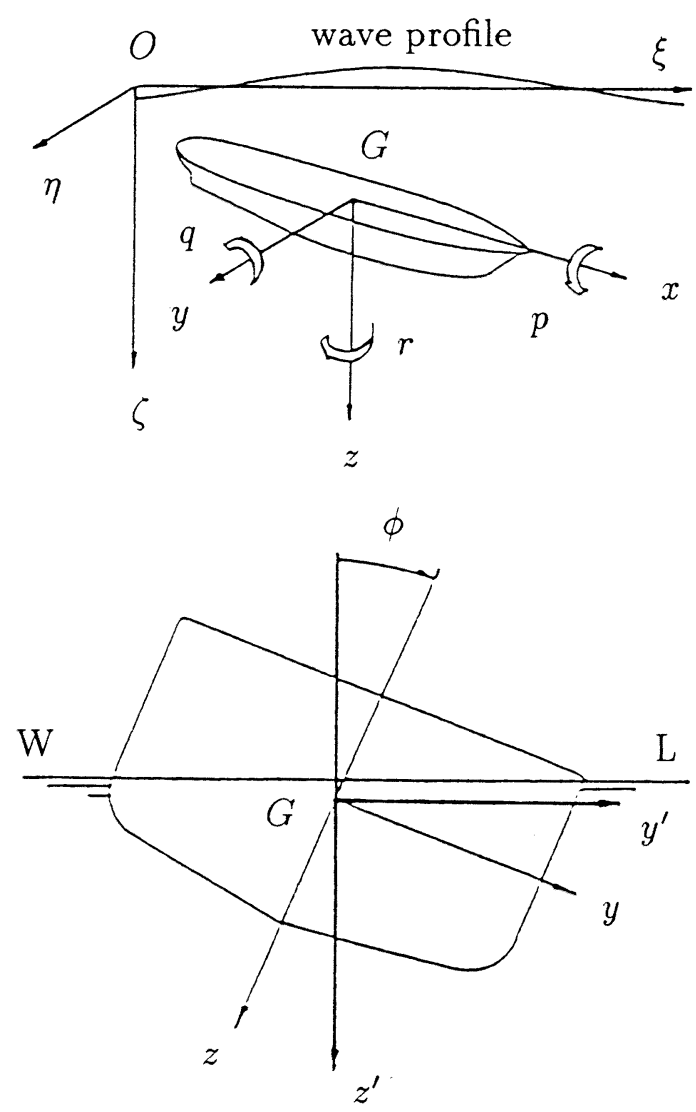

Fig. 1 Earth axes $O-\xi, \eta, \zeta$, General body axes $G-x$, $y, z$ and Horizontal body axes $G-x, y^{\prime}, z^{\prime}$

where $p$ is the pressure over the entire immersed surface of the ship, and $V$ is the immersed volume of the ship. The hydrostatic pressure $p$ including that of a sinusoidal waves $\zeta_{\omega}$

$$
\zeta_{\omega}=a \cos k(\xi-c t)
$$

at any time $t$ and position $\xi$ in the earth axes is given by

$$
p=\rho g \zeta-\rho g a e^{-k \zeta} \cos k(\xi-c t)
$$

where $\rho$ is the density of water, $k$ is the wave number, $a$ is the amplitude of the waves and $c$ is the phase velocity of the wave. By using the following transformation set between the earth and body axes

$$
\begin{aligned}
\xi-\xi_{G}= & x \cos \phi \cos \theta \\
& +y(\cos \phi \sin \theta \sin \phi-\sin \phi \cos \phi) \\
& +z(\cos \psi \sin \theta \cos \phi+\sin \phi \sin \phi) \\
\cong & x \cos \phi-(y \cos \phi-z \sin \phi) \sin \phi \\
\zeta-\zeta_{G}= & -x \sin \theta+y \cos \theta \sin \phi+z \cos \theta \cos \phi \\
\cong & -x \theta+y \sin \phi+z \cos \phi
\end{aligned}
$$

the pressure $p$ in the body axes is given as

$$
\begin{aligned}
& p=\rho g\left(\zeta_{G}-x \theta+y \sin \phi+z \cos \phi\right) \\
& -\rho g a e^{-k\left(\zeta_{G}-x \theta+y \sin \phi+z \cos \phi\right)} \\
& \times \cos k\left[\xi_{G}+x \cos \psi-(y \cos \phi-z \sin \phi) \sin \psi\right. \\
& -c t]
\end{aligned}
$$

where $\zeta_{G}$ is the sinkage, $\theta$ the trim angle, $\phi$ the heel angle and $\psi$ the heading angle of ship to waves. Accordingly pressure gradients in body axes are given respectively as

$$
\begin{aligned}
& \left(\frac{\partial p}{\partial x}\right)=-\rho g \theta-\rho g a k \theta e^{-k\left(\zeta_{G}-x \theta+y \sin \phi+z \cos \phi\right)} \\
& \times \cos k\left[\xi_{G}+x \cos \psi\right. \\
& -(y \cos \phi-z \sin \phi) \sin \phi-c t] \\
& +\rho g a k \cos \phi e^{-k\left(\zeta_{c}-x \theta+y \sin \phi+z \cos \phi\right)} \\
& \times \sin k\left[\xi_{G}+x \cos \psi\right. \\
& -(y \cos \phi-z \sin \phi) \sin \phi-c t] \\
& \left(\frac{\partial p}{\partial y}\right)=\rho g \sin \phi+\rho g a k \sin \phi e^{-k\left(\zeta_{c}-x \theta+y \sin \phi+z \cos \phi\right)} \\
& \times \cos k\left[\xi_{G}+x \cos \psi\right. \\
& -(y \cos \phi-z \sin \phi) \sin \phi-c t] \\
& -\rho g a k \cos \phi \sin \phi e^{-k\left(\zeta_{c}-x \theta+y \sin \phi+z \cos \phi\right)} \\
& \times \sin k\left[\xi_{G}+x \cos \psi\right. \\
& -(y \cos \phi-z \sin \phi) \sin \phi-c t] \\
& \left(\frac{\partial p}{\partial z}\right)=\rho g \cos \phi+\rho g a k \cos \phi e^{-k\left(\zeta_{G}-x \theta+y \sin \phi+z \cos \phi\right)} \\
& \times \cos k\left[\xi_{G}+x \cos \psi\right. \\
& -(y \cos \phi-z \sin \phi) \sin \phi-c t] \\
& +\rho g a k \sin \phi \sin \phi e^{-k\left(\zeta_{C}-x \theta+y \sin \phi+z \cos \phi\right)} \\
& \times \sin k\left[\xi_{G}+x \cos \psi\right. \\
& -(y \cos \phi-z \sin \phi) \sin \phi-c t]
\end{aligned}
$$

By introducing Horizontal body axes coordinate system $G-x, y^{\prime}, z^{\prime}$ which are related to the body axes coordinate system as

$$
\begin{aligned}
& y^{\prime}=y \cos \phi-z \sin \phi \\
& z^{\prime}=z \cos \phi+y \sin \phi
\end{aligned}
$$

and also the pressure gradients in Horizontal body axes are

$$
\begin{aligned}
& \left(\frac{\partial p}{\partial y^{\prime}}\right)=\left(\frac{\partial p}{\partial y}\right) \cos \phi-\left(\frac{\partial p}{\partial z}\right) \sin \phi \\
& \left(\frac{\partial p}{\partial z^{\prime}}\right)=\left(\frac{\partial p}{\partial z}\right) \cos \phi+\left(\frac{\partial p}{\partial y}\right) \sin \phi
\end{aligned}
$$

Substituting $(\partial p / \partial y)$ and $(\partial p / \partial z)$ in Eq. ( 7$)$ into Eq. ( 9 ), the pressure gradients in Horizontal body axes are obtained in the form simplified as

$$
\begin{aligned}
& \left(\frac{\partial p}{\partial x}\right) \cong-\rho g \theta+\rho g a k e^{-k\left(\zeta_{c}-x \theta+z^{\prime}\right)} \cos \psi \sin k\left(\xi_{G}\right. \\
& \left.+x \cos \psi-y^{\prime} \sin \psi-c t\right) \\
& \left(\frac{\partial p}{\partial y^{\prime}}\right)=-\rho g a k e^{-k\left(\zeta_{G}-x \theta+z^{\prime}\right)} \sin \psi \sin k\left(\xi_{G}\right. \\
& \left.+x \cos \psi-y^{\prime} \sin \psi-c t\right) \\
& \left(\frac{\partial p}{\partial z^{\prime}}\right)=\rho g+\rho g a k e^{-k\left(\zeta_{G}-x \theta+z^{\prime}\right)} \cos k\left(\xi_{G}\right. \\
& \left.+x \cos \psi-y^{\prime} \sin \psi-c t\right)
\end{aligned}
$$

In the same way, Froude-Krylov forces and moments in Horizontal body axes are described in the form :

$$
\begin{aligned}
& X_{(F . K)}=-\iiint_{V} \frac{\partial p}{\partial x} d V \\
& Y_{(F, K)}^{\prime}=Y_{(F . K)} \cos \phi-Z_{(F . K)} \sin \phi=-\iiint_{V} \frac{\partial p}{\partial y^{\prime}} d V \\
& Z_{(F, K)}^{\prime}=Z_{(F . K)} \cos \phi+Y_{(F . K)} \sin \phi=-\iiint_{V} \frac{\partial p}{\partial z^{\prime}} d V \\
& K_{(F . K)}=-\iiint_{V}\left\{\left(\frac{\partial p}{\partial z^{\prime}}\right) y^{\prime}-\left(\frac{\partial p}{\partial y^{\prime}}\right) z^{\prime}\right\} d V
\end{aligned}
$$




$$
M_{(F, K)}^{\prime}=M_{(F, K)} \cos \phi-N_{(F, K)} \sin \phi \cong \iiint_{V}\left(\frac{\partial p}{\partial z^{\prime}}\right) x d V
$$

$$
N_{(F . K)}^{\prime}=N_{(F . K)} \cos \phi+M_{(F . K)} \sin \phi \cong-\iiint_{V}\left(\frac{\partial p}{\partial y^{\prime}}\right) x d V
$$

Evaluation of Froude-Krylov forces

Substituting the first equation in Eq. (10) into Eq. (11), Froude-Krylov force $X_{(F . K)}$ is given in the form:

$$
\begin{aligned}
X_{(F . K)}= & \rho g \theta \int_{L} A(x) d x \\
& -\rho g a k \cos \psi \int_{L} e^{-k\left(\zeta_{G}-x \theta\right)} \\
& \times \sin k\left(\xi_{G}+x \cos \psi-c t\right) d x \\
& \times \iint_{A} e^{-k z^{\prime}} \cos \left(k y^{\prime} \sin \psi\right) d A \\
& +\rho g a k \cos \psi \int_{L} e^{-k\left(\zeta_{G}-x \theta\right)} \\
& \times \cos k\left(\xi_{G}+x \cos \psi-c t\right) d x \\
& \times \iint_{A} e^{-k z^{\prime}} \sin \left(k y^{\prime} \sin \psi\right) d A
\end{aligned}
$$

where $A(x)$ is the sectional area of instantaneously immersed volume and $L$ the ship length. The integral with respect to the sectional area $A(x)$ can be described as

$$
\begin{aligned}
& \int_{z_{w}^{\prime}}^{z_{d}^{\prime}} e^{-k z^{\prime}} d z^{\prime} \int_{y_{p}^{\prime}}^{y_{s}^{\prime}} \cos \left(k y^{\prime} \sin \psi\right) d y^{\prime} \\
& =\int_{z^{\prime} w}^{z^{\prime} d} \frac{\sin \left(k y_{s}^{\prime} \sin \psi\right)-\sin \left(k y_{p}^{\prime} \sin \psi\right)}{k \sin \psi} e^{-k z^{\prime}} d z^{\prime}
\end{aligned}
$$

where $y_{p}^{\prime}$ and $y_{s}^{\prime}$ are the coordinates of wave surface on the port and starboard sides of the ship, $z_{w}^{\prime}$ and $z_{d}^{\prime}$ are the coordinates of wave surface in the immersed section and the immersed draft of the ship in waves. Unfortunately, it is not so easy to obtain an analytical solution of the integral with respect to $z^{\prime}$ because $y_{p}^{\prime}$ and $z_{p}^{\prime}$ are a function of $z^{\prime}$ in the direction of depth. So that it has to be considered how to evaluate to this integral. Since Eq. (14) means the integral of local pressure gradient with respect to the sectional area, the average pressure gradient in the section would be equal to the ratio of the integral to the sectional area. And then the average pressure gradient in a heeled condition will be nearly equal to that in the upright condition of the ship in a wave. If so, the average value of Eq. (14) will be obtained from that of the equivalent barge section at the upright condition as follows :

$$
\begin{aligned}
& \text { Average value of Eq. ( } 14)=\frac{\sin \left(k \frac{B(x)}{2} \sin \psi\right)}{k \frac{B(x)}{2} \sin \psi} \\
& \times e^{-k \bar{d}(x)}
\end{aligned}
$$

where the breadth $B(x)$ of equivalent barge section is equal to that of the actual ship section, the sectional area $A(x)$ of barge is the same as that of the actual ship and the draft $\bar{d}(x)$ of equivalent barge section is determined to be the same sectional area as that of the actual ship. Using the average value, the integral of Eq. (13) can be evaluated as

$$
\iint_{A} e^{-k z^{\prime}} \cos \left(k y^{\prime} \sin \psi\right) d A=\frac{\sin \left(k \frac{B(x)}{2} \sin \psi\right)}{k \frac{B(x)}{2} \sin \psi}
$$

$$
\times e^{-k \bar{d}(x)} A(x)
$$

and

$$
\iint_{A} e^{-k z^{\prime}} \sin \left(k y^{\prime} \sin \psi\right) d A=0
$$

According to Eq. (16) and Eq. (17), Froude-Krylov forces $X_{(F, K)}$ and also in the same way, $Y_{(F, K)}^{\prime}$ and $Z_{(F, K)}^{\prime}$ can be described in the following practical formulas:

$$
\begin{aligned}
X_{(F . K)} \cong & \rho g \theta \int_{L} A(x) d x \\
& -\rho g a k \cos \psi \int_{L} \frac{\sin \left(k \frac{B(x)}{2} \sin \psi\right)}{k \frac{B(x)}{2} \sin \psi} \\
& \times e^{-k\left(\zeta_{C}-x \theta+\bar{d}(x)\right)} A(x) \\
& \times \sin k\left(\xi_{G}+x \cos \psi-c t\right) d x \\
Y_{[F . K)}^{\prime} \cong & \rho g a k \sin \psi \int_{L} \frac{\sin \left(k \frac{B(x)}{2} \sin \psi\right)}{k \frac{B(x)}{2} \sin \psi} \\
& \times e^{-k\left(\zeta_{G}-x \theta+\bar{d}(x)\right) A(x)} \\
& \times \sin k\left(\xi_{G}+x \cos \psi-c t\right) d x \\
Z_{(F . K)}^{\prime} \cong & -\rho g \int_{L} A(x) d x \\
& -\rho g a k \int_{L} \frac{\sin \left(k \frac{B(x)}{2} \sin \psi\right)}{k \frac{B(x)}{2} \sin \psi} \\
& \times e^{-k\left(\zeta_{C}-x \theta+\bar{d}(x)\right)} A(x) \\
& \times \cos k\left(\xi_{G}+x \cos \psi-c t\right) d x
\end{aligned}
$$

where $X_{(F, K)}$ and $Z_{(F, K)}^{\prime}$ consist of two kinds of force due to the pressure gradients of still water and that of waves caused by Smith effect. The imersed sectional area $A(x)$ has to be determined at the balanced condition with respect to the ship displacement and moment about the center of gravity. The balanced condition will be discussed later in this paper.

\section{Evaluation of Froude-Krylov moments}

Substituting the second and third equations in Eq. (10) into the first equation in Eq. (12), $K_{(F . K)}$ is given in the forms:

$$
\begin{aligned}
K_{(F . K)}= & -\rho g \int_{L} d x \iint_{A} y^{\prime} d A \\
& -\rho g a k \int_{L} e^{-k\left(\zeta_{C}-x \theta\right)} \\
& \times \cos k\left(\xi_{G}+x \cos \psi-c t\right) d x \\
& \times \iint_{A} e^{-k z^{\prime}} y^{\prime} \cos \left(k y^{\prime} \sin \psi\right) d A \\
& -\rho g a k \int_{L} e^{-k\left(\zeta_{C}-x \theta\right)} \\
& \times \sin k\left(\xi_{G}+x \cos \psi-c t\right) d x \\
& \times \iint_{A} e^{-k z^{\prime}} y^{\prime} \sin \left(k y^{\prime} \sin \psi\right) d A \\
& -\rho g a k \sin \psi \int_{L} e^{-k\left(\zeta_{C}-x \theta\right)} \\
& \times \sin k\left(\xi_{G}+x \cos \psi-c t\right) d x \\
& \times \iint_{A} e^{-k z^{\prime}} z^{\prime} \cos \left(k y^{\prime} \sin \psi\right) d A \\
& +\rho g a k \sin \psi \int_{L} e^{-k\left(\zeta_{C}-x \theta\right)} \\
& \times \cos k\left(\xi_{G}+x \cos \psi-c t\right) d x
\end{aligned}
$$




$$
\times \iint_{A} e^{-k z^{\prime}} z^{\prime} \sin \left(k y^{\prime} \sin \psi\right) d A
$$

Here, the integral with respect to the sectional area $A(x)$ can be described as

$$
\begin{aligned}
& \int_{z_{w}}^{z^{\prime} d} e^{-k z^{\prime}} d z^{\prime} \int_{y^{\prime} p}^{y^{\prime} s} y^{\prime} \cos \left(k y^{\prime} \sin \psi\right) d y^{\prime} \\
& \quad=\int_{z^{\prime} w}^{z^{\prime} d} \frac{1}{k^{2} \sin ^{2} \phi}\left\{\left[y_{s}^{\prime} \sin \left(k y_{s}^{\prime} \sin \psi\right)\right.\right. \\
& \left.\quad-y_{p}^{\prime} \sin \left(k y_{p}^{\prime} \sin \psi\right)\right] k \sin \psi \\
& \left.\quad+\cos \left(k y_{s}^{\prime} \sin \psi\right)-\cos \left(k y_{p}^{\prime} \sin \psi\right)\right\} e^{-k z^{\prime}} d z^{\prime} \\
& \cong \int_{z^{\prime} w}^{z^{\prime} d}\left(y_{s}^{\prime 2}-y_{p}^{\prime 2}\right) e^{-k z^{\prime}} d z^{\prime} \\
& \cong 0
\end{aligned}
$$

and

$$
\begin{aligned}
& \int_{z_{w}^{\prime}{ }_{w}^{z_{d}}}^{z^{\prime}} e^{-k z^{\prime}} d z^{\prime} \int_{y_{\rho}^{\prime}}^{y_{s}^{\prime}} y^{\prime} \sin \left(k y^{\prime} \sin \psi\right) d y^{\prime} \\
& \quad=\int_{z^{\prime} w}^{z^{\prime} d} \frac{1}{k^{2} \sin ^{2} \phi}\left\{\sin \left(k y_{s}^{\prime} \sin \psi\right)-\sin \left(k y_{p}^{\prime} \sin \psi\right)\right.
\end{aligned}
$$

$$
\begin{aligned}
& -\left[y_{s}^{\prime} \cos \left(k y_{s}^{\prime} \sin \psi\right)\right. \\
& \left.\left.-y_{p}^{\prime} \cos \left(k y_{p}^{\prime} \sin \psi\right)\right] k \sin \psi\right\} e^{-k z^{\prime}} d z^{\prime} \\
& \cong 0
\end{aligned}
$$

where $\left(y_{s}^{\prime 2}-y_{p}^{\prime 2}\right)$ will be considered to be nearly equal to zero. Since the ship breadth is very small compared with wave length, it would be acceptable to make the approximation as

$$
\begin{aligned}
& \sin \left(k y_{s}^{\prime} \sin \psi\right) \cong k y_{s}^{\prime} \sin \psi \\
& \cos \left(k y_{s}^{\prime} \sin \psi\right) \cong 1
\end{aligned}
$$

According to Eq. ( 16 ), ( 17 ), ( 20 ) and (21), FroudeKrylov moments $K_{(F, K)}, M_{(F, K)}^{\prime}$ and $N_{(F, K)}^{\prime}$ can be described in the following practical formulas:

$$
\begin{aligned}
K_{(F . K)} \cong & -\rho g \int_{L} y_{B(x)}^{\prime} A(x) d x \\
& -\rho g a k \sin \psi \int_{L} \frac{\sin \left(k \frac{B(x)}{2} \sin \psi\right)}{k \frac{B(x)}{2} \sin \psi} \\
& \times e^{-k\left(\zeta_{G}-x \theta+\bar{d}(x)\right)} z_{B(x)}^{\prime} A(x) \\
& \times \sin k\left(\xi_{G}+x \cos \psi-c t\right) d x \\
M_{(F . K)}^{\prime} \cong & \rho g \int_{L} x A(x) d x \\
& +\rho g a k \int_{L} \frac{\sin \left(k \frac{B(x)}{2} \sin \psi\right)}{k \frac{B(x)}{2} \sin \psi} \\
& \times e^{-k\left(\zeta_{C}-x \theta+\bar{d}(x)\right)} x A(x) \\
& \times \cos k\left(\xi_{G}+x \cos \psi-c t\right) d x \\
N_{(F . K)}^{\prime} \cong & \rho g a k \int_{L} \sin \psi \frac{\sin \left(k \frac{B(x)}{2} \sin \psi\right)}{k \frac{B(x)}{2} \sin \psi} \\
& \times e^{-k\left(\zeta_{C}-x \theta+\bar{d}(x)\right)} x A(x) \\
& \times \sin k\left(\xi_{G}+x \cos \psi-c t\right) d x
\end{aligned}
$$

where $y_{B}^{\prime}(x)$ and $z_{B}^{\prime}(x)$ are the center of immersed sectional area $A(x)$ and given as

$$
y_{B(x)}^{\prime}=\frac{\iint_{A} y^{\prime} d A}{\iint_{A} d A}, z_{B(x)}^{\prime}=\frac{\iint_{A} z^{\prime} d A}{\iint_{A} d A}
$$

Determination of immersed sectional area
When the ship is displaced in a quartering sea, the ship weight $W$ has to be balanced with the buoyancy $Z_{(F, K)}^{\prime}$, the trim moment $M_{(F, K)}^{\prime}$ and heel moment $K_{(F, K)}$ have to be zero without the hydrodynamic forces and moments acting on the immersed hull surface. In such a hydrostatic balanced condition, the sinkage $\zeta_{G}$, heel angle $\phi$ and trim angle $\theta$ are given as the solution of the following equation:

$$
\begin{aligned}
& Z_{(F . K)}^{\prime}+W=0 \\
& M_{(F, K)}^{\prime}=0 \\
& K_{(F, K)}=0
\end{aligned}
$$

However, it is impossible to find the solution by the same way as the linearized theory because the immersed sectional area $A(x)$ is a function of $\zeta_{G}, \phi$ and $\theta$. So that the solution will be found by using a step-by-step computing manner.

Now, assuming that the coordiates of wave surfce are given by $\left(y_{p}, z_{p}\right)$ and $\left(y_{s}, z_{s}\right)$ on the port and starboard sides of the ship displaced with $\zeta_{G}, \phi$ and $\theta$, the relation among them can be obtained by putting $\zeta=\zeta_{w}$ into Eq. (5). That is

$$
\begin{aligned}
& \zeta_{w}\left(x, y_{p}, z_{p}\right)=\zeta_{G}-x \theta+y_{p} \sin \phi+z_{p} \cos \phi \\
& \zeta_{w}\left(x, y_{s}, z_{s}\right)=\zeta_{G}-x \theta+y_{s} \sin \phi+z_{s} \cos \phi
\end{aligned}
$$

And also the wave surface $\zeta_{w}(x, y, z)$ can be obtained from substituting Eq. ( 5 ) into Eq. ( 3 ). Since

$$
\begin{aligned}
\zeta_{w}(x, y, z)= & a \cos k\left[\xi_{G}+x \cos \psi\right. \\
& -(y \cos \phi-z \sin \phi) \sin \psi]
\end{aligned}
$$

Eq. (26) can be written as

$$
\begin{aligned}
& a \cos k\left[\xi_{G}+x \cos \psi-\left(y_{p} \cos \phi-z_{p} \sin \phi\right) \sin \phi\right] \\
& \quad=\zeta_{G}-x \theta+y_{p} \sin \phi+z_{p} \cos \phi \\
& a \cos k\left[\xi_{G}+x \cos \phi-\left(y_{s} \cos \phi-z_{s} \sin \phi\right) \sin \phi\right] \\
& \quad=\zeta_{G}-x \theta+y_{s} \sin \phi+z_{s} \cos \phi
\end{aligned}
$$

When the values of $\zeta_{G}, \phi$ and $\theta$ are given, one has to find out the coordinates $\left(y_{p}^{\prime}, z_{p}^{\prime}\right)$ and $\left(y_{s}^{\prime}, z_{s}^{\prime}\right)$ to satisfy the condition of Eq. (28). Since the immersed sectional area $A(x)$ is a function of the given values of $\zeta_{G}, \phi$ and $\theta$, the solution of Eq. (25) which satisfies the condition of Eq. (28) is obtained by using an iterative manner.

\section{Evaluation of restoring force}

When the ship is displaced in a wave, the restoring force of the ship may vary with respect to the relative position of ship to waves. As well known, the righting lever $G Z$ of the ship in a wave may be reduced at a wave crest amidship compared with that in a still water and the reduction is not so small for the ship of the same length as a wave. A practical method to compute the reduction will be presented here to investigate the effect of restoring force on the ship motion and capsizing in severe following and quartering seas. The righting lever $G Z$ against an external heel moment can be defined by the relation $W \cdot G Z=-K_{(F, K)}$ since $K_{(F, K)}$ is the heel moment about the center of gravity of the ship, according to the first equation in Eq. ( 23 )

$$
W \cdot G Z=\rho g \int_{L} y_{B(x)}^{\prime} A(x) d x
$$




$$
\begin{aligned}
& +\rho g a k \sin \psi \int_{L} \frac{\sin \left(k \frac{B(x)}{2} \sin \psi\right)}{k \frac{B(x)}{2} \sin \psi} \\
& \times e^{-k\left(\zeta_{G}-x \theta+\bar{d}(x)\right)} z_{B(x)}^{\prime} A(x) \\
& \times \sin k\left(\xi_{G}+x \cos \psi-c t\right) d x
\end{aligned}
$$

The first term of Eq. (29) indicates the restoring force based on the same concept as that in a still water and the pressure gradient is equal to that in a still water. That will be why the so-called Smith effect is neglected for computing the restoring force in a following sea. The second one is the restoring force coming from the different concept that is based on the side force $Y_{(F . K)}^{\prime}$ acting on the center of buoyancy $z_{B}^{\prime}$. For the ship displaced in the condition with the given heel angle corresponding to a external force, the balanced conditions of the sinkage $\zeta_{G}$ and trim angle $\theta$ will be

$$
\begin{aligned}
Z_{(F, K)}^{\prime}+W & =0 \\
M_{(F, K)}^{\prime} & =0
\end{aligned}
$$

and also Eq. (30) can be solved by using the same way as mentioned before. Therefore, the restoring force at any given angle is obtained from Eq. (29).

\section{Critical situations leading up to capsize and the simulations}

The ship motions and capsizing in severe following and quartering seas are characterized by the several parameters such as wave height and length, the relative velocity of ship to waves, the metacentric height, the initial position of ship to waves, the heading angle, the reduction of restoring force and the natural frequency of ship, and so on. Then the unfavorable combination of them may induce the ship to the critical situations as to whether the ship capsizes. In the previous paper 22), free running model experiments were carried out for investigations into extreme rolling of ships in a critical situation. One of the problems with this approah is that even if capsizes do occur it is difficult to reproduce the same situations in order to investigate the parameters involved or check the effect of a change in parameters. It may be the benefit for this problem to use a mathematical model which can carry out time domain numerical simulations at a considerable level correspoding to model experiments and, if possible, it had better to be described in the six degrees of freedom because the critical situations may consist of several directions of motion and the parameters. From such a point of view, one of the authors proposed a mathematical model with respect to Horizontal body axes in the previous papers, the result was briefly acceptable in comparison with the results of model experiments. The equations of motion are

$$
\begin{aligned}
& m(\dot{U}-V \dot{\phi}+W \dot{\theta})=X-m g \theta+T(1-t)-R \\
& m(\dot{V}+U \dot{\psi})=Y^{\prime} \\
& m(\dot{W}-U \dot{\theta})=Z^{\prime}+m g \\
& I_{x x} \ddot{\phi}=K \\
&\left(I_{y y} \cos ^{2} \phi+I_{z z} \sin ^{2} \phi\right) \ddot{\theta}+I_{x x} \dot{\phi} \dot{\psi}=M^{\prime}
\end{aligned}
$$

$$
\left(I_{z z} \cos ^{2} \phi+I_{y y} \sin ^{2} \phi\right) \ddot{\phi}-I_{x x} \dot{\theta} \dot{\phi}=N^{\prime}
$$

The hydrostatic and hydrodynamic forces and moments are

$$
\begin{aligned}
& X=X_{(F, K)}+X \text { (Hydrodynamic forces) } \\
& Y^{\prime}=Y_{(F, K)}^{\prime}+Y^{\prime} \text { (Hydrodynamic forces) } \\
& Z^{\prime}=Z_{(F, K)}^{\prime}+Z^{\prime} \text { (Hydrodynamic forces) } \\
& K=K_{(F, K)}+K \text { (Hydrodynamic moments) } \\
& M^{\prime}=M_{(F, K)}^{\prime}+M^{\prime} \text { (Hydrodynamic moments) } \\
& N^{\prime}=N_{(F, K)}^{\prime}+N^{\prime} \text { (Hydrodynamic moments) }
\end{aligned}
$$

In Eq. (33) and (34), the hydrodynamic forces and moments due to the velocity and acceleration of motion both ship and waves will be dependent on the frequency and amplitude of ship motion. The frequenucy dependence can be characterized in motion for a linear and high frequency region but not for a nonlinear and low frequency region at present. However, it will be tried to obtain the hydrodynamic forces from an approximate evaluation to be available at present. The hydrodynamic forces and moments about horizontal motion are evaluated by using Motora's chart 4 ) with repect to the added masses and moments of inertia and Inoue's practical formulas 14) with respect to the stability derivatives of sway and yaw motions. On the other hand, the hydrodynamic forces and moments about vertical motions are evaluated by using Tasai's practical formulas 7 ) in which the hydrodynamic coefficients are specified at the natural frequency of heave and pitch in linearized motion. The damping coefficient of rolling is evaluated by Takahashi's practical formulas 9 ) including the effect of ship speed. The mentioned above formulas are already written in the previous paper 23) of this study. The equations of motion are solved in the time domain by using a step-by-step method based on Runge-Kutta.

In this final paper, the authors would like to draw out some critical situations as to whether the ship capsizes by using the aid of numerical simulation programs. The attentions in this paper will be concentrated into the critical situations for the effect of the following important parameters.

\section{Effect of wave height}

Effect of relative velocity of ship to waves

Effect of metacentric height

Effect of initial position of ship to waves

Effect of heading angle of ship to waves

Numerical simulations were carried out for the 4990 GT

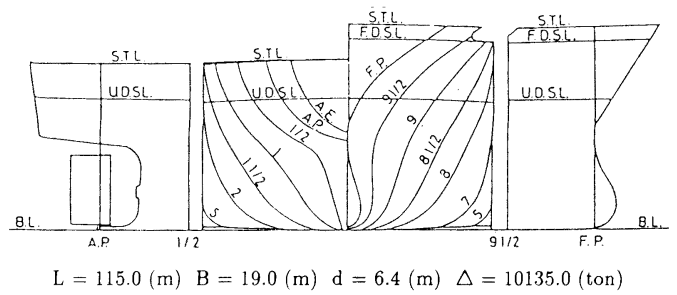

Fig. 2 Principal dimension 


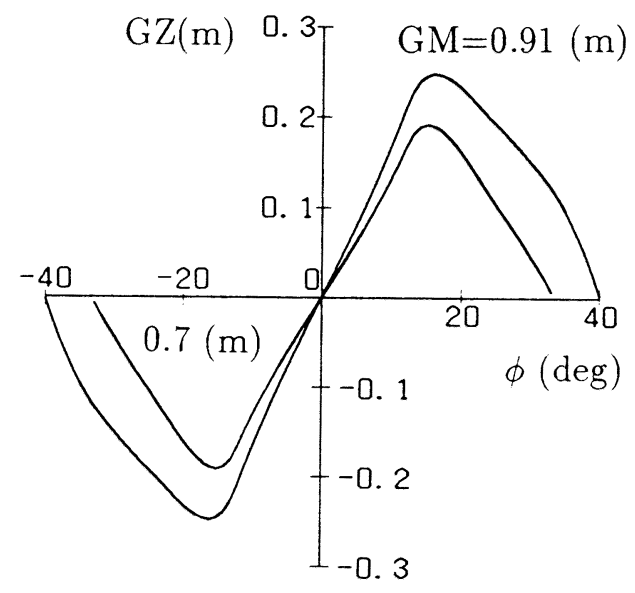

Fig. $3 G Z$ curve in still water

class container ship of which body plan and principal particulars are shown in Fig. 2. The righting levers GZ in still water are shown in Fig. 3.

\section{Effect of wave height}

Four examples of the numerical simulation are carried out to check the effect of a change in wave height and the results are shown in Fig. 4 where the condition of ship and waves are defined as follows:

ratio of wave and ship length, $\lambda / L=1$

ratio of wave height and length,

$H / \lambda=0.04,0.0467,0.047,0.05$

heading angle of ship to waves, $\psi=0(\mathrm{deg})$

initial position of ship to waves, $\xi_{c} / \lambda=0$ relative velocity of ship to waves, $U / c=0.6$

metacentric height, $G M=0.7(\mathrm{~m})$

In Fig. 4, the relative position of ship to waves is defined by $\xi_{G} / \lambda=0$ at the wave trough amidship, $\xi_{G} / \lambda= \pm 0.5$ at the wave crest amidship and $\xi_{G} / \lambda= \pm 1$ at the position where it comes to the wave trough again. The positive sign stands for the ship velocity larger than the wave velocity and the negative sign for the ship velocity smaller than the wave velocity. In the examples, the ship is initially forced in an inclined condition at 5 degrees and it is released to be free during computation. The first example in Fig. 4 stands for a rolling which is quickly damped out at the waves of $H / \lambda=0.04$ and next two examples show that a successive rolling of parametric resonance characterized by a Mathieu equation occurs at the waves $H / \lambda=0.0467$ and 0.047 . This seems to be a critical situation with respect to the effect of wave height. In the last example, the ship is eventually capsized in the waves of $H / \lambda=0.05$.

\section{Effect of relative velocity of ship to waves}

Four examples are shown to check the effect of a change in the relative velocity of ship to waves $U / C=$ $0.5,0.55,0.65,0.7$ where the ratio of other parameters are the same as them mentioned above. The first example in Fig. 5 stands for a rolling which is gradually damped out at a slightly higher frequency than the natural roll frequency. The second one shows a successive rolling of parametric resonance and the third one stands for a rolling which is irregularly damped out at a slightly lower frequency than the natural roll frequency. In the last one, the ship capsizes at a relative velocity $U / c=0.7$. Three examples in Fig. 5 stand for a
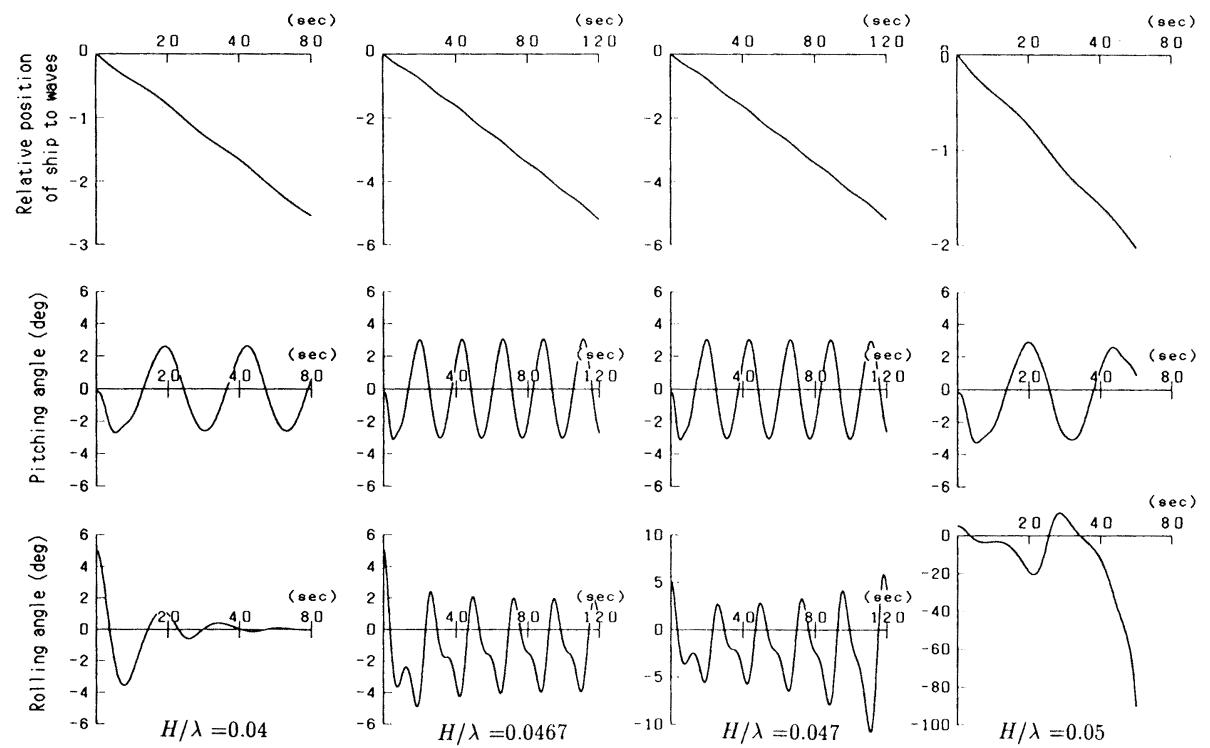

Fig. 4 Time domain simulation for a change of wave height 

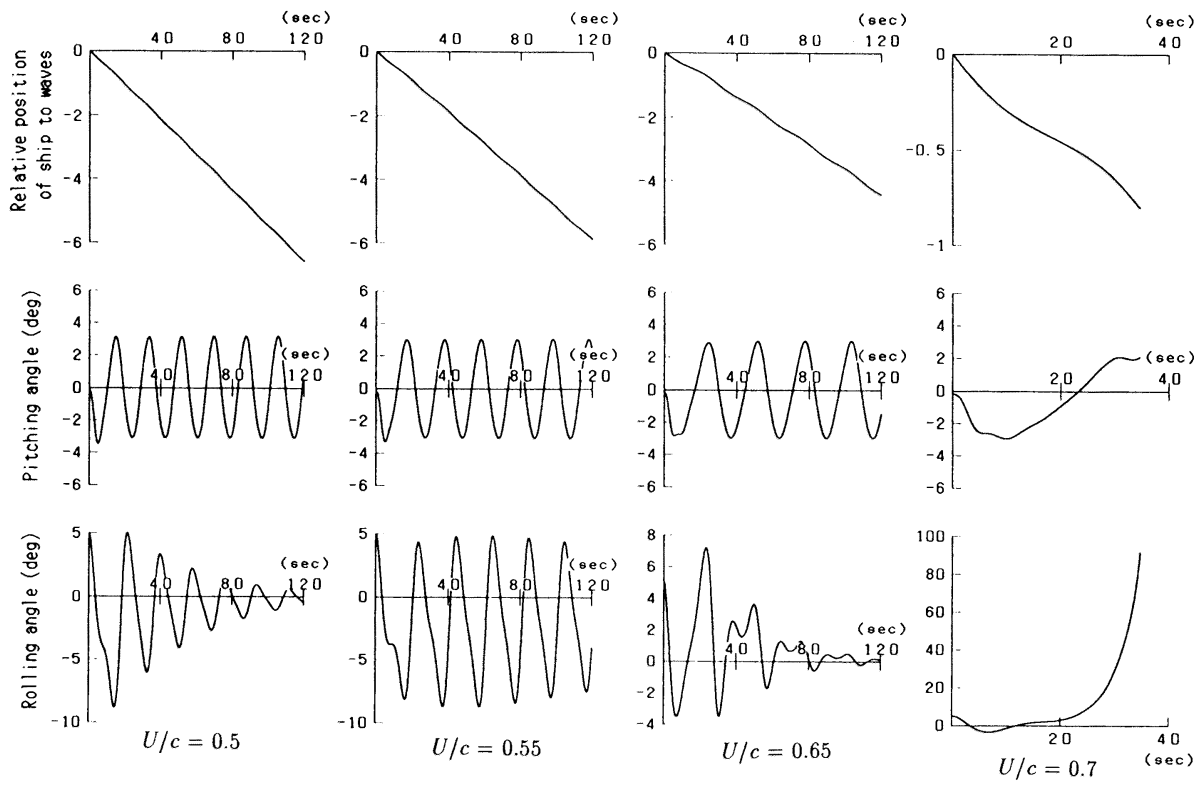

Fig. 5 Time domain simulation for a change of rela tive velocity of ship to waves
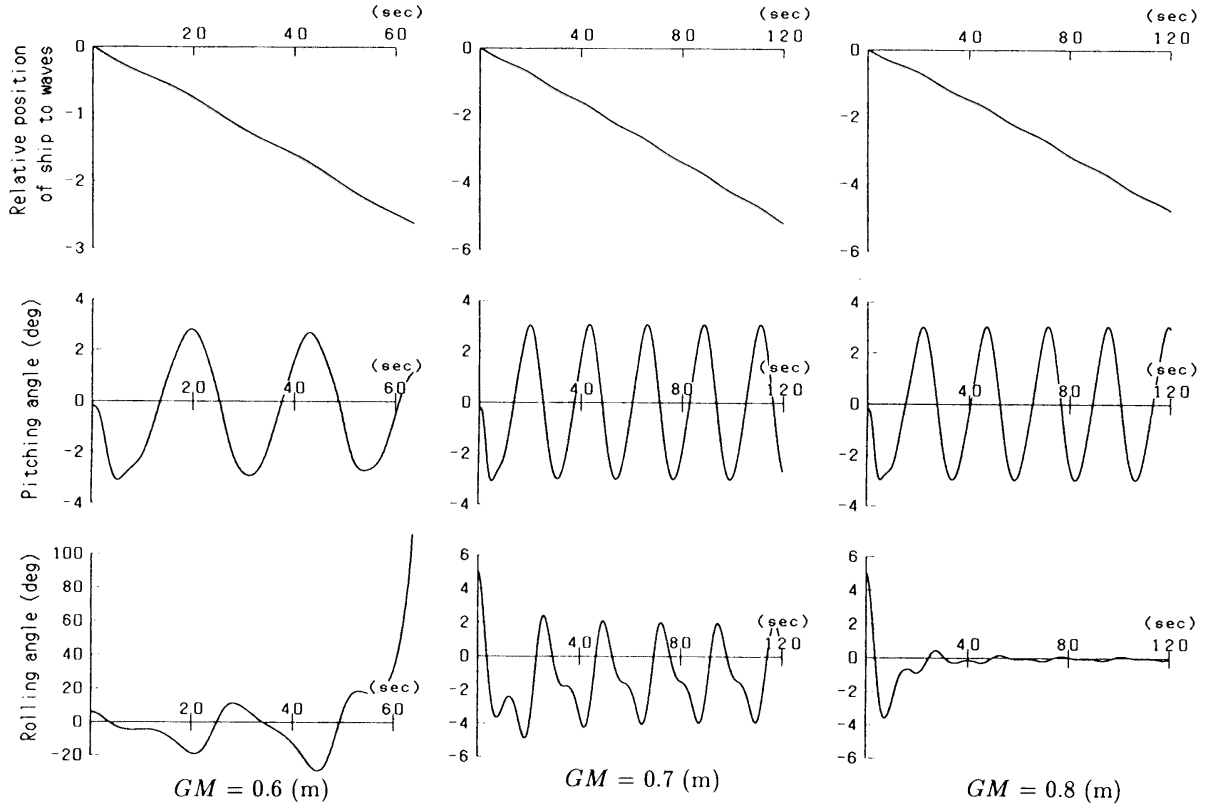

Fig. 6 Time domain simulation for a change of metacentric height

typical property of the parametric resonance. In the critical situation where the ship is running with a successive rolling, the speed up may induce the ship to take the capsize caused by pure loss of stability, and the speed down may reduce the amplitude of rolling untill that is damped out.

\section{Effect of metacentric height}

Fig. 6 shows the simulation results for three examples of the different metacentric height $G M=0.6 \mathrm{~m}, 0.7 \mathrm{~m}$ and $0.8 \mathrm{~m}$ in which the ratio of wave height and length is $H / \lambda=0.0467$ and the other parameters are the same as them mentioned above. The first example stands for $G M=0.6 \mathrm{~m}$. Though the stability index required by IMO Resolution A562 is equal to 1.3, the ship capsizes in this condition. The secod one shows a critical condition in a 

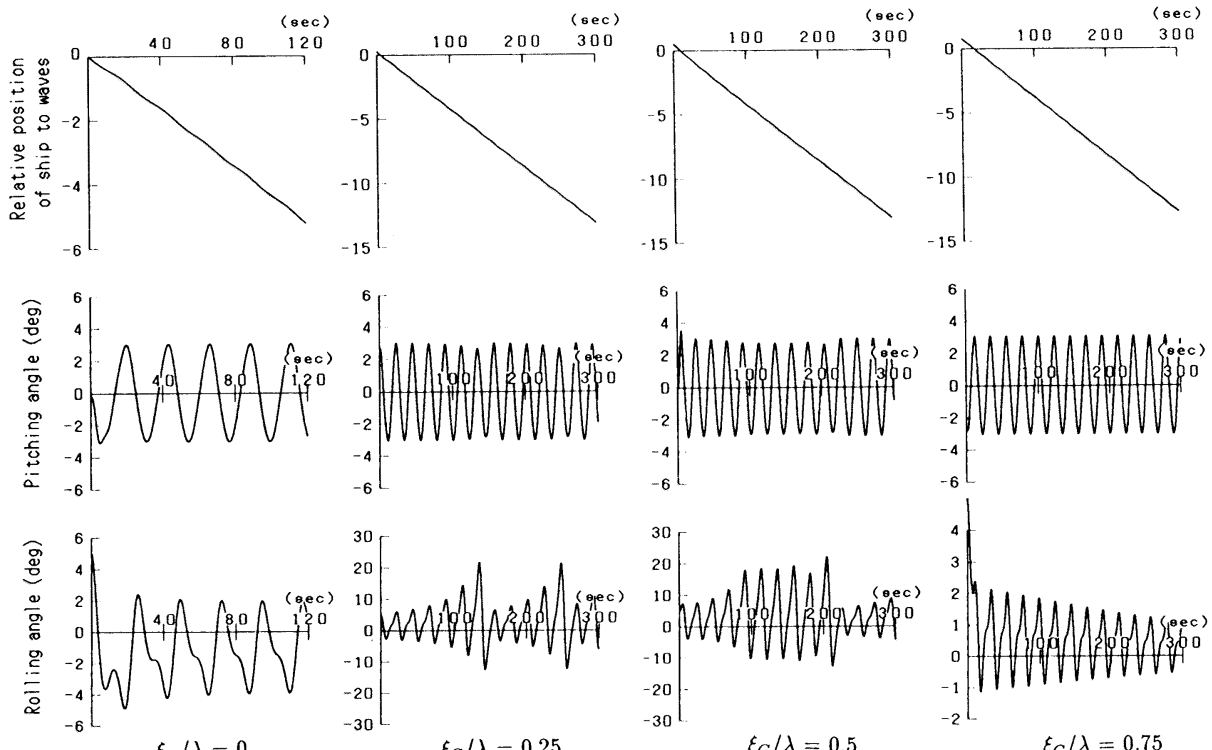

$\xi_{G} / \lambda=0$

$\xi_{G} / \lambda=0.25$

$\xi_{G} / \lambda=0.5$

$\xi_{G} / \lambda=0.75$

Fig. 7 Time domain simulation for a change of initial position of ship to waves
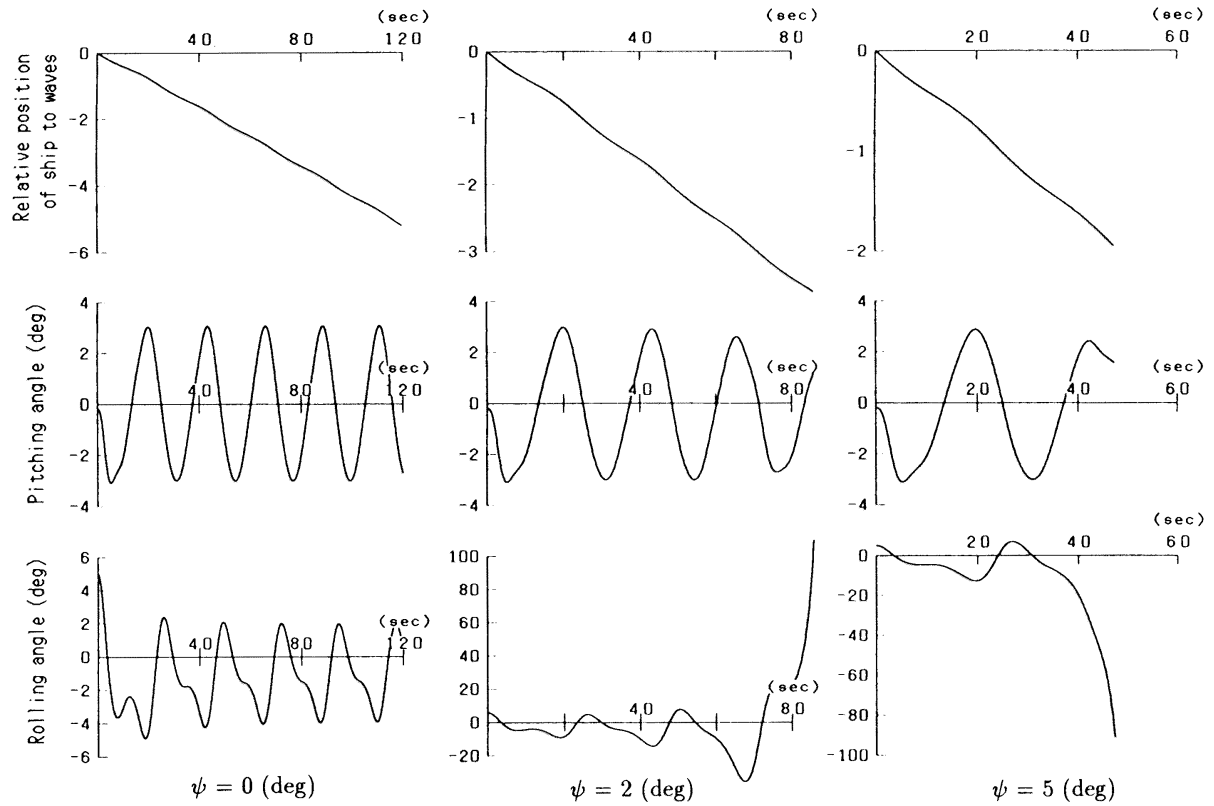

Fig. 8 Time domain simulation for a change of heading angle of ship to waves

change of metacenteric height. In the last one, the rolling is quickly damped out.

\section{Effect of initial position of ship to waves}

Fig. ( 7 ) shows four examples to check the effect of a change in the initial position where the ship is released to be free at the wave trough amidship $\zeta_{G} / \lambda=0$, at the up slope amidship $\xi_{G} / \lambda=0.25$, at the wave crest amidship $\xi_{G} / \lambda=0.5$ and at the down slope amidship $\xi_{c} / \lambda=0.75$. Though the rollings in the first and last examples are not developed to large angle, the rolling in the second and third examples are irregularly developed into a considerably large angle. 
Effect of heading angle of ship to waves

Finally, Fig. ( 8 ) shows three examples to check the effect of a change in heading angle of ship to waves $\psi$ $=0,2,5$ degrees and the other parameters are the same as them mentioned above. the first example stands for a successive rolling of parametric resonance mentioned before. The most pronounced rolling and capsizes occur when the ship has a small heading angle $\psi=2,5$ degrees. At present it is somewhat hard for a large heading angle to investigate this effect in detail since this effect is concerned with the so-called broaching which requires more reasonable evaluation of hydrodynamic forces and moments acting on the ship in severe quartering seas.

\section{Conclusions}

This paper shows a practical method for simulating ship motions and capsizing in severe following seas. In order to point out a critical situation leading up to capsize for a container ship, the authors carried out a number of numerical simulations to check the effect of a change in parameters by using the aid of simulation program based on a new mathematical model. All of the examples in this numerical simulation are carried out for the ship in the waves about the length of the ship. So that the wave profile causes a change in righting force and this gives rise to a parametric resonance as characterized by a Mathieu equation. The natural frequency of roll $\omega_{\phi}$ is equal to $0.352 / \mathrm{sec}$ and the encounter frequency of ship to waves are over the range of frequency $0.22<\omega_{e}<0.37$. A successive rolling of parametric resonance occurs at a range of frequency $1.07<\omega_{\phi} / \omega_{e}<1.20$, at a larger range than the frequency $\omega_{\phi} / \omega_{e}>1.20$ and at a range of the frequency $1.07<\omega_{\phi} / \omega_{e}<1.38$ as shown in Fig. 5. When the wave height is slightly increased, a successive rolling are gradually developed in the amplitude. Furthermore the capsize occurs in the more than higher waves as shown in Fig. 4. Though the critical situations leading up to capsize are affected by several parameters mentioned in this paper, the controllable parameters for captains will be ship speed and heading angle of ship to waves. The critical speed can be determined from natural frequency of roll and encounter velocity of ship to waves. The captain can avoid the critical speed. These informations about the critical situation leading up to capsize will be available to discuss the maneouvring manual and the criteria against capsizing of ship in severe following seas. It is concluded that this simulation method can be used as a useful tool for investigation into the sensitivity of parameters with respect to critical situations of ship in severe following seas. As the next step, the radiation and diffraction problem with respect to the hydrodynamic forces and moments must be considered in detail.

Model experiments and numerical simulations are carried out under RR24 Research Panel of the Ship
Building Assocation of Japan and also this research is partly supported by Grant-in-Aid for Scientific Research of the Ministry of Education. (Project Number 02302055)

\section{References}

1) Watanabe, Y., On the Dynamic Properties of the Transverse Instability of a ship due to Pitchings, J. of Soc. of Naval Arch., Vol. 53, 1934.

2) Grim, O., Rollschwingungen, Stabilitat und Sicherheit im seegang, Schiffistechnik, 1952.

3) Kerwin, J.E., Notes on roll in longitudinal Waves, I. S. P., Vol. 2 (16 ), 1955.

4) Motora, S., On the Measurement of Added Mass and Added Moment of Ineria for Ship Motion, J. of Soc. of Naval Arch., Vol. 105-107, 1959, 1960.

5) Paulling, J.R., The Transverse Stability of a Ship in a Longitudinal Seaway, J. of Ship Research, SNAME, Vol. 4, No. 4, March, 1961.

6) Dr-Ing. O. Grim, Beitrag zu dem problem der Sicherheit des Schiffes in Seegang, Schiff und hafen, helt $6,1961$.

7) Tasai, F., Damping Force and Added Mass of Ships Heavng and Pitching (Continued), Trans. of the West-Japan Soc. of Naval Arch., Vol. 21, 1961.

8) Du Cane, P., Goodrich, gG. J., The Following Sea, Broaching and Surging, Trans. RINA, Vol. 104, April, 1962.

9) Takahashi, T., Mechanism of Rolling and Application, (in Japanese), Report of Mitsubishi Heavy Industry Nagasaki Technical Institute, No. 2842, 1960, Unpublished

10) Boses, P., Steuern eines Shiffes im Schweren Achterlichen Seegange, J. S. T. G., Bd, 1971

11) Price, W. G., The Stability of Ship in a Simple Sinusoidal Wave, J. of Mechanical Engineering Science, Vol. 14, 1972.

12) Chou, S. J., Oakely, O. H.,, Paulling, J. R., Van Slyke, R., Wood, P. D., Zink, P. F., Ship Motions and Capsizing in Astern Seas, Dep. of Transportation United States Coast Guard, Rep. No. CGD-103-75, 1974.

13) Paulling, J. R., Oakley, and Wood, P. A."Ship Capsizing in Heavy Seas: The Correlation of Theory and Experiments". International Conferece on Stability of Ships and Ocean Vehicles. University of Strathclyde, 1975

14) Inoue, S., Kijima, K. and Moriyama, F., "Presumption of Hydrodynamic Derivatives on Ship Manoeuvring in Trimmed Condition" Trans. of West-Japan Society of Naval Architects. No. 55, March 1978.

15) Renilson, M. R.,, Driscoll, A., Broaching-An Investigation into the Loss of Directional Control in Severe Following Seas, Spring Meeting RINA, 1981.

16) Motora, S., Fujino, M., Fuwa, T., On the Mechanism of Broaching-to Phenomena, STABILITY'82, 1982.

17) Hamamoto, M., Nomoto, K., Transverse Stability of Ship in a Following Sea, STABILITY'82, 1982.

18) Kuo, C., Vassalos, D., The Stratchclyde Approach to Practical Ship Stability Criteria, Workshop on Stability, Germanischrr Lloyd, 1984 
19) Hamamoto, M., Transverse Stability of Ship in a Quartering Sea, STABILITY'86, 1986.

20) Ohkusu, M., Prediction of Wave Forces in a Ship Running in a Following Waves with Very Low Encounter Frequency, J. of Soc. of Naval Arch. Vol. 159, 1986.

21) Prediction of Ship Manoeuverability and its Applications, 4th Marine Dynamics Symposium, 1987.

22) Hamamoto, M., Akiyoshi, T., Study on Ship Motions and Capsizing in Following Seas (1st
Report), J. of Soc. of Naval Arch. Vol. 163, 1988.

23) Hamamoto, M., Shirai, T., Study on Ship Motions and Capsizing in Following Seas (2nd Report), J. of Soc. of Naval Arch. Vol. 165, 1989.

24) Jan O. de Kat, J. Randolph Paulling, The Simulation of Ship Motions and Capsizing in Severe Seas, The Society of Naval Architects and Marine Engineers, 1989.

25) Hamamoto, M., Shirai, T., Wakiyama, N., An Analytical Approach to Capsizing of a Ship in Following Seas, STABILITY'90, 1990. 